\section{Loss of heparan sulphate proteoglycan and retinal microinfarcts in diabetes mellitus}

\begin{abstract}
Dear Sir,
We read with interest the paper by Deckert et al. on albuminuria as an indicator of widespread vascular damage in Type 1 (insulin-dependent) diabetes mellitus [1]. In their paper, Deckert et al. suggest that the loss of normal heparan sulphate proteoglycan in the endothelial cell membranes of retinal capillaries may contribute to the formation of microthrombi and/or platelet plugs. Some of our work supports this hypothesis. We have reported that retinal cotton wool spots - thought to be retinal microinfarcts [2] - may be found in early diabetic retinopathy [3]. This has now been acknowledged by other investigators [4]. This observation led us to investigate in vivo coagulation in diabetic patients. We found that plasma fibrinopeptide A increased significantly with increasing severity of diabetic retinopathy [5]. The postulated loss of endothelial cell proteoglycan [1] may contribute to the activation of the blood coagulation factors in such diabetic patients. However, longitudinal studies of diabetic patients are needed in order to determine whether this activation of the coagulation system is the cause or the result of vascular disease in diabetes mellitus.
\end{abstract}

Yours sincerely,

M. S. Roy and M.E.Rick

\section{References}

1. Deckert T, Feldt-Rasmussen B, Borch-Johnsen K, Jensen T, Kofoed-Enevoldsen A (1989) Albuminuria reflects widespread vascular damage. The Steno hypothesis. Diabetologia 32: 219-226

2. Kohner EM, Dollery CT, Bulpitt CJ (1969) Cotton wool spots in diabetic retinopathy. Diabetes 18: 691-704

3. Roy MS, Rick ME, Higgins KE, McCulloch JC (1986) Retinal cotton-wool spots: an early finding in diabetic retinopathy? $\mathrm{Br} \mathrm{J}$ Ophthalmol 70: 772-778

4. Klein R, Klein B, Moss SE, Davis MD, De Mets DL (1989) The Wisconsin Epidemiology Study of Diabetic Retinopathy. IX: Four year incidence and progression of diabetic retinopathy when age at diagnosis is less than 30 years. Arch Ophthalmol 107:237-247

5. Roy MS, Rick M, Podgor M (1988) Plasma fibrinopeptide A, betathromboglobulin and platelet factor 4 in diabetic retinopathy. Invest Ophthalmol Vis Sci 29:856-860

\section{Dr. M. Roy}

National Institutes of Health

National Eye Institute and Clinical Pathology Department

9000 Rockville Pike

Bethesda, MD 20892

USA

\section{Abnormal blood rheology and diabetic nephropathy}

\footnotetext{
Dear Sir,

Deckert et al. [1] implied that diabetic albuminuria has a unique mechanism, a conclusion reached only by ignoring large sections of the relevant literature. Studies which interpret glomerular function in terms of one or two plasma proteins in the urine do not provide information about total glomerular permeability. Spectra of proteins in diabetic urine can be demonstrated by sodium dodecyl sulfate - polyacrylamide gel electrophoresis but the implications of such information is lost on those who focus on albumin excretion. Further-
}

more, as similar protein spectra can be shown in post-exertional urine from healthy subjects there is no need to postulate damage to or injury of the glomerular filter to explain proteinuria [2] but the level of proteinuria will be increased if the efficiency of tubular reabsorption is impaired.

Abnormal blood rheology impairs microcirculatory blood flow and influences glomerular filtration adversely. As the haemorheological changes will be enhanced by glomerular filtration, blood flow rate in the peritubular plexus will be reduced, thus hindering tubular reabsorption. For these reasons the most surprising and illogical aspect of Deckert et al.'s paper [1] was the lack of recognition that blood is involved in the genesis of albuminuria and that blood is different after glomerular filtration. In addition there was a total disregard for those reports which show that diabetic blood rheology is abnormal.

Glomerular filtration results in haemoconcentration which persists until rehydration occurs in the peri-tubular plexus and when there is pre-existing hyperviscosity (as in diabetes) this abnormality will be exaggerated by glomerular filtration to the detriment of postglomerular blood flow. Other conditions involving blood hyperviscosity such as polycythaemia and cyanotic heart disease also have an associated proteinuria [3].

Deckert et al. [1] wrote that "Increased blood pressure ... is not the cause of albuminuria," and "Nor does increased intraglomerular pressure seem to be the cause of albuminuria." In their opinion "... genetically determined alterations in the composition of the extracellular matrix" is the cause of persistent proteinuria. In view of the evidence to the contrary it is surprising that they concluded that "... these changes seem to be severe enough to induce changes in permeability," in diabetic patients with a albuminuria but Baba et al. [4] noted that "...2 of 10 microalbuminuric subjects were normoalbuminuric when supine." In addition the consequences of microalbuminuria in nondiabetic subjects has been described; treatment with anti-hypertensive agents reduces levels of albuminuria; while diabetic kidneys grafted into non-diabetic subjects functioned normally and failed to show the functional changes expected from the postulated, genetically-determined defect in basement membrane permeability. By proposing compositional changes in the glomerular basement membrane (GBM) as the cause of increaed permeability Deckert et al. ignored the fact that it is the physical nature of the GBM, including the anionic charge layer which determines its permeability [5].

Temporary excretion of high molecular weight plasma proteins demonstrable in post-exertional urine shows that normal glomeruli have the capacity to filter large proteins without damage [2]. Such observations can be explained in terms of the pressure-dependent permeability of GBM's as a consequence of their biological thixotropy [6]. Molecules traversing the GBM must have sufficient kinetic energy to override the anionic charge layer (for negatively charged molecules) and to cause deformation of the GBM lattice. Subsequently there is a time-related reformation which restores the integrity of the lattice. For these reasons larger, positively charged molecules filter at similar pressures to those which are smaller but negatively charged.

Although the concept of basement membrane thixotropy has not gained general acceptance there has been a change in position by the proponents of the pore theory. A "haemodynamic" concept invoking "elevated pressures and flows" has appeared but the concept provides no physical basis for the glomerular filter which could explain temporary proteinuria nor does it incorporate recognised haemorheologic information.

The resistance to flow of hyperviscous blood in the peritubular plexus will be an important determinant of intraglomerular pressure and therefore of the hyperfiltration associated with increased blood viscosity [7]. Reports of direct relationships between blood viscosity and proteinuria in diabetic [8] and in non-diabetic subjects [9] are of particular significance as the only effect of increased blood viscosity will be to stimulate a rise in intravascular pressure sufficient to sustain flow of the viscous blood. When pentoxifylline was used to improve blood rheology in diabetics patients with retinopathy [10] not only was there a fall in blood pressure with improvement in the retinal microcirculatory disorders but also there was a marked reduction in proteinuria and in the albumin excretion rate. 